\title{
Use of image correlation to measure hygric swelling in rocks
}

Ferrer, Belén, Tomás, M. Baralida, Benavente, David, Mas, David

Belén Ferrer, M. Baralida Tomás, David Benavente, David Mas, "Use of image correlation to measure hygric swelling in rocks," Proc. SPIE 11353, Optics, Photonics and Digital Technologies for Imaging Applications VI, 113531T (1 April 2020); doi: 10.1117/12.2555077

SPIE. Event: SPIE Photonics Europe, 2020, Online Only, France 


\title{
Use of image correlation to measure hygric swelling in rocks
}

\author{
Belén Ferrer*a , M. Baralida Tomás ${ }^{\mathrm{a}}$, David Benavente ${ }^{\mathrm{b}}$, David Mas ${ }^{\mathrm{a}}$ \\ anst. of Physics Applied to the Sciences and Technologies, Univ. Alicante P.O. Box, 99, 03080 \\ Alicante, Spain; ${ }^{b}$ Department of Earth and Environmental Sciences, University of Alicante, Spain
}

\begin{abstract}
Some materials undergo an hygric expansion when they are soaked. In porous rocks, this effect is enhanced by the pore space that allows the water to reach every part of its volume and to hydrate the most of their swelling parts. This enlargement has negative structural consequences in the vicinity since adjacent elements will support some compressions or displacements. Recently image-based methods have arisen in this field due to their advantages versus traditional methods. Among all image processing methods, digital image correlation (DIC) is one of the most used in all areas.

In this work, we propose a new methodology based on DIC for the calculation of the hygric expansion of materials. We use porous sandstone, with dimensions $14 \times 14 \times 30 \mathrm{~mm}$ to measure its hygric swelling using an industrial digital camera and a telecentric objective. We took one image every 5 minutes to characterize the whole swelling process. Due the large magnification, the whole $14 \mathrm{~mm}$ length of one contour was not in the image and therefore we lost the image scale reference. To solve this, a 1951 USAF test was used to calibrate the imagen. The telecentric objective and a narrow deep of field allowed to have the specimen surface exactly on the same plane that the USAF test was during the calibration. The image was pointed to one corner of the specimen, to obtain information not only of its vertical displacement due to its expansion but also of its horizontal movement. Preliminary results show that the proposed methodology provides reliable information of the hygric swelling using a non-contact methodology, with an accuracy of 1 micron.
\end{abstract}

Keywords: Hygric rock swelling, digital image correlation, own surface texture

\section{INTRODUCTION}

Measurement of rock shrinkage and swelling by the presence of water is a key parameter to analyze the behavior of rocks. For historical buildings, as their structures are made by these rocks, the shrinkage measurement of the rock becomes part of its structural health monitoring. Most common technique to measure rock swelling is using transducers sensors, such as Linear Variable Differential Transformer (LVDT) [1] or cell loads [2]. However, as they are contact methods, they can modify the behavior of the rock, especially for small samples. With LVDT the laboratory setups for measuring vertical displacements of the rock contour are easy to do, but measurement of horizontal displacement makes complex setups. Additionally, these sensors typically have an accuracy of 1 micron, which can be not enough for some applications.

Recently, image-based methods have arisen in this field due to its advantages over traditional methods. Among all methods, digital image correlation (DIC) is one of the most used in all areas. In swelling measurement, DIC has been used for different materials, such as sandstone [3], wood [4] or argillaceous rock [5], for example. Regarding the setup, commercial and industrial cameras are usually used, although some very small specimens require an environmental scanning electron microscope [5]. When using industrial cameras, some integrated software is also used to perform DIC, such the ARAMIS package [6]. However, the use of such software imposes a previous sample preparation that is not always possible. DIC has been heavily tested on pseudo-speckle textures, which can be obtained by painting the sample surface with a uniform color and then, adding small dots with another contrasted color by spraying it [7]. Nevertheless, sometimes the surface preparation is not possible due to its size or its composition, since the specimen could be damaged by the paint, or even because it is part of a historical building and its swelling on site is trying to be measured. On these cases, the use of the natural surface texture to perform DIC is highly recommendable. This possibility basically relies on the imaging system and its configuration, since rock surface could have a pseudospeckle-like texture similar to speckle depending only on the magnification and the type of material. This hypothesis was tested in [8], where a DIC measurement of a concrete test specimen under loading-unloading cycles below its yield limit was done using only the concrete surface texture.

Optics, Photonics and Digital Technologies for Imaging Applications VI, edited by Peter Schelkens, Tomasz Kozacki, Proc. of SPIE Vol. 11353, 113531T · @ 2020 SPIE CCC code: $0277-786 X / 20 / \$ 21 \cdot$ doi: $10.1117 / 12.2555077$ 
On this work we employ a prismatic sandstone specimen with $14 \times 14 \times 30 \mathrm{~mm}$ to measure its hygric swelling using its own texture. To do that, a proper image magnification should be done in order to have a texture suitable to perform DIC with a good accuracy. That magnification gives some issues that were solved in this work.

\section{MATERIAL AND METHODS}

\subsection{Experimental setup}

In this paper, we tested a porous sandstone used as construction and building material. It consists of quartz and feldspar with a clay-rich matrix. It presents a connected porosity in the range of 10-12\%. That rock was cut in samples with a size of $14 \times 14 \times 30 \mathrm{~mm}$. Samples were placed in a container and covered with water until a 1/3 of their height. Thus, water moved by capillarity forces to the top of the samples as a piston-like imbibition process.

Images of the rock surface were taken by a color camera Basler acA4600-10uc, with a resolution of 4608x3288px. The telecentric objective Myutron VTL0513, with the additional lens VTL05FC was used. This objective together with the lens provided a magnification of of $0.65 \mathrm{x}$. This magnification, with a working distance of $50 \mathrm{~mm}$ (figure 1), gives a clear image of the rock's components, with a size of $300 \mathrm{px}^{2}$ per component approximately (figure 2). Additionally, on this rock its components have different colors which results in a particular texture that seems to be suitable for DIC calculations. The optical system was centered in an upper corner of the probe so that movements in vertical and horizontal movements could be registered.

Setup optimization for obtaining a clear image of the texture has the disadvantage of losing the view of total length of the piece under study, even of its shorter size. Therefore, the size of the sample cannot be used as a scale reference an alternative procedure should be used instead in order to obtain the ratio $\mathrm{px} / \mathrm{mm}$. To do that, we took advantage of the telecentric objective used. Technical specifications for this objective give a depth of field among 1.68 and $0.47 \mathrm{~mm}$. For the magnification used in this work, the working depth of field is approximately $1 \mathrm{~mm}$. With the magnification fixed, we used a USAF 1951in the object plane in order to obtain the image scale, resulting in a ratio of $1.32 \mathrm{px} / \mathrm{micron}$ (figure 3 ).

Once the movement is measured, the hygric swelling is obtained through the engineering normal strain, which is the change in length per unit of the original length in the considered direction. A positive normal strain means a swelling and a negative one a shrinkage. In order to avoid errors due to misalignment, the camera was horizontally adjusted with a tri-wheel leveling base with bubble level bags (figure 4).

A small container was used to partially submerge the rock probe inside the water until a predetermined level. A syringe was used to pour the water into the container, as a way to avoid movement of the rock once its position has been adjusted to have a focused image (figures 1 and 4). An image every 5 minutes was taken using this setup.

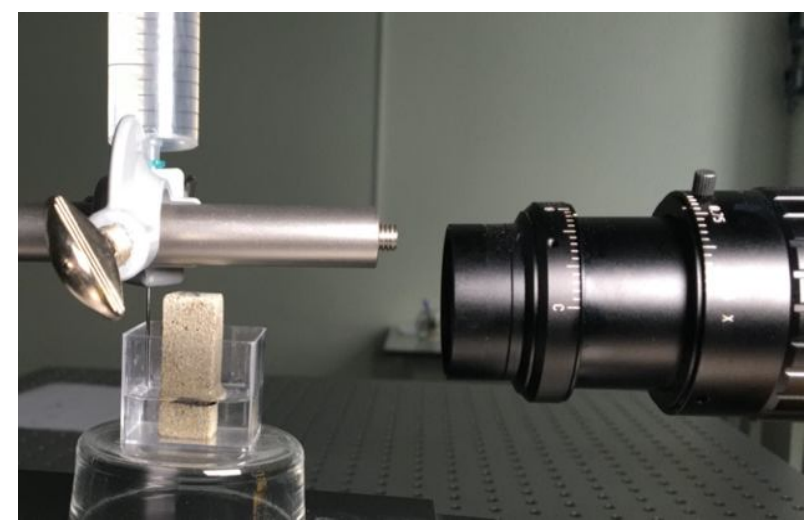

Figure 1. Telecentric objective and setup for the probe with a working distance of $50 \mathrm{~mm}$. The syringe used to add the water can be seen in the background part of the picture. 


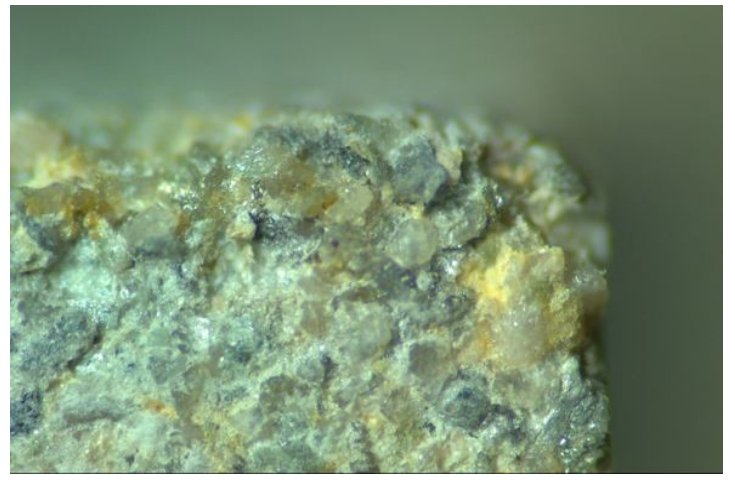

Figure 2. A corner of the rock probe, in the final setup configuration that gives the best image texture to calculate movements by DIC.

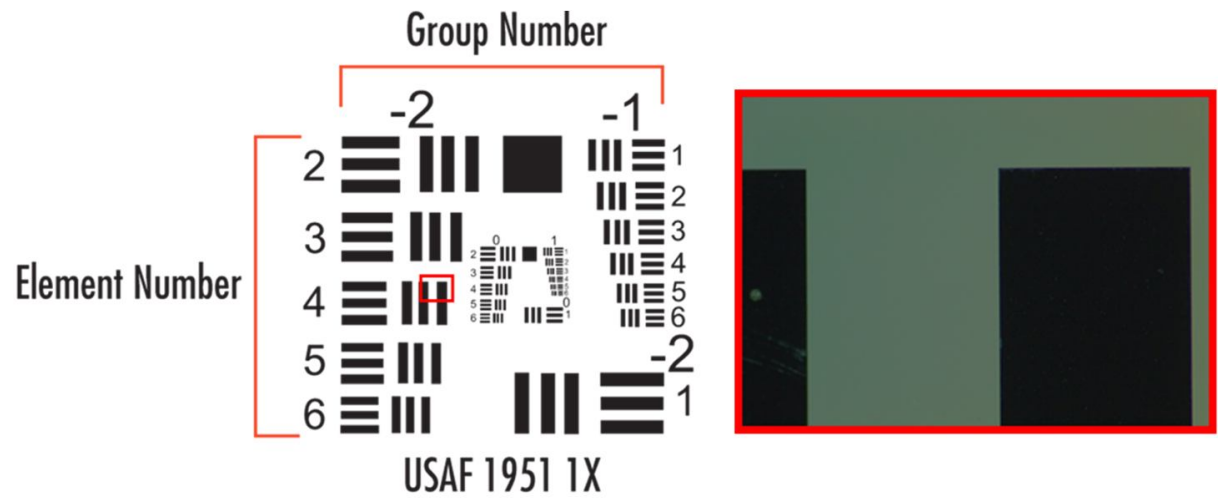

Figure 3. USAF test used for camera calibration (left). Image taken. Image taken from camera corresponding to the red rectangle in USAF test (right).

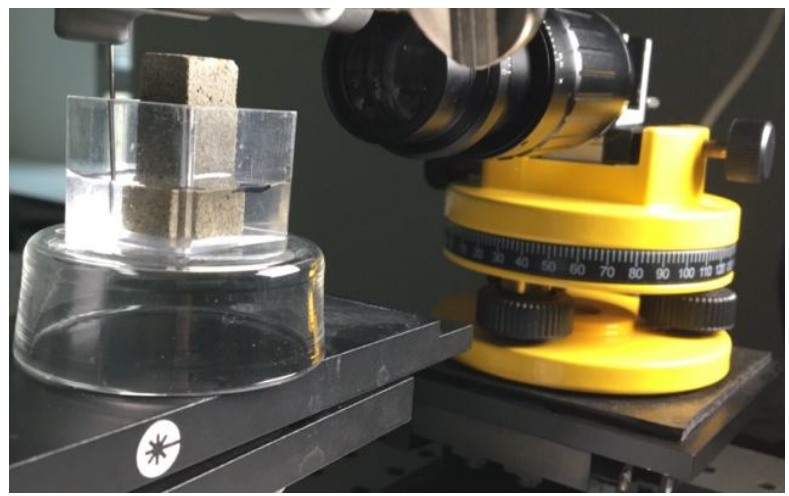

Figure 4. Tri-wheel leveling base with bubble level bags used to have the imaging system horizontally levelled.

\subsection{Image processing}

The procedure followed to obtain movement measurement is based in DIC. A normalized cross correlation is done using the first image as reference for all the rest of images. That calculation is done only inside of a region of interest (ROI) that was previously selected and cropped in all frames for the same sequence, with an approximate size of $2000 \times 2000$ px. Having into account that the biggest grain size roughly is 300x300, that means that at least 6 big grains are inside the ROI, besides other smaller particles that may also contribute to the correlation peak. As the final expected movement is small (some microns, that means only some pixels), the ROI was fixed and taken large enough to contain the moving pattern through all the sequence. Template size for the correlation is also related to the expected movement and to the ROI size 
since the maximum coincidence should lie inside the ROI. A template $50 \mathrm{px}$ smaller in both dimensions than the ROI was taken. Finally, to increase the performance of the method and obtain subpixel accuracy, a neighborhood of $3 \times 3 \mathrm{px}$ around the correlation peak is selected and fitted to a quadratic surface. Then, the maximum of the peak is reevaluated. Figure 6 shows a flow chart with the main steps used to find the movement. The procedure is fully described in [8].

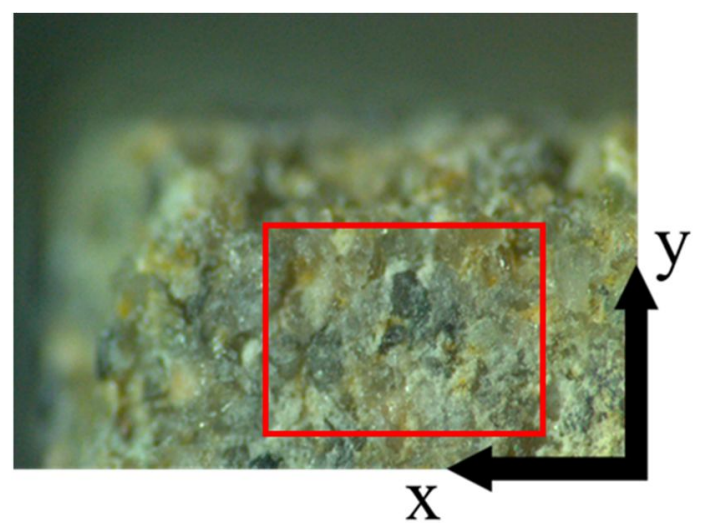

Figure 5. Corner recorded in all samples to track movements, ROI selected to perform DIC for sample C2 and origin and positive sense of movements for the calculations done here.

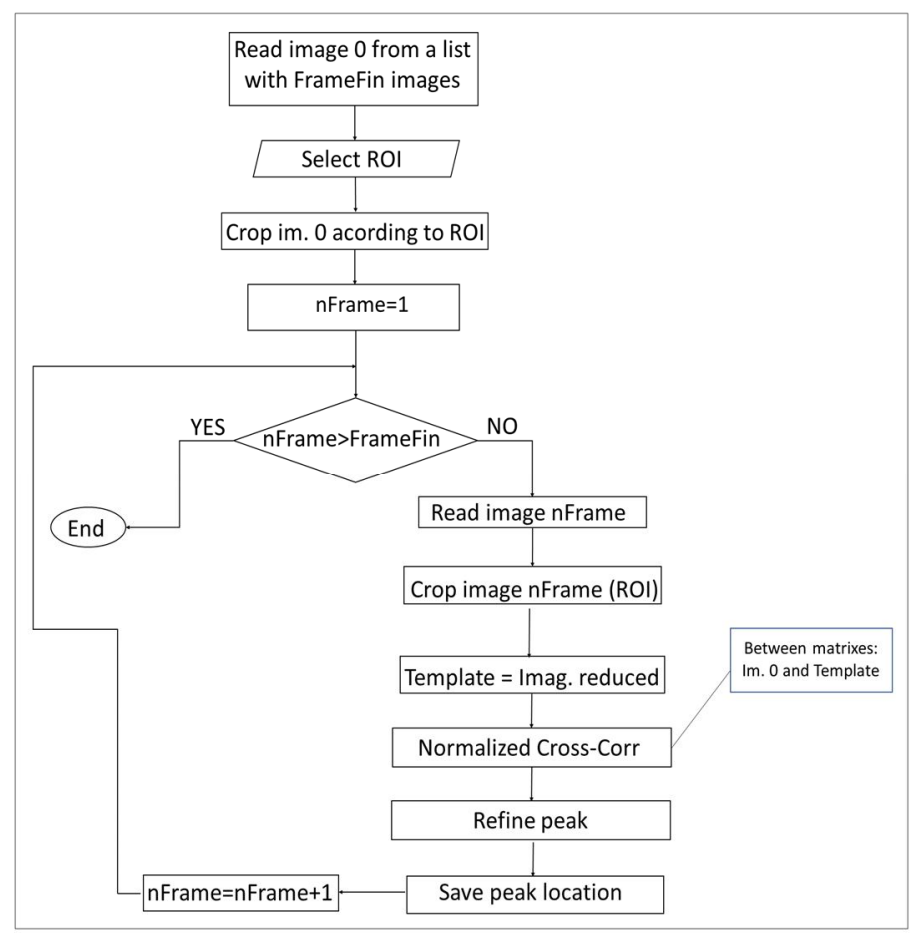

Figure 6. Flow chart with the main steps of the procedure.

\section{RESULTS}

Vertical and horizontal movement were determined by DIC, following the procedure described in the previous section. Results for both movements and for the four samples are shown in figure 5. In all cases, first vertical displacement (figure 
7 (left)) is sharply sloped due to the quick swelling of the bottom part of the probe that is submerged in water, which makes the stone to dilate very fast. This behavior has an inflection point at approximately 0.25 hours for all samples after which the slope is smoother. This is probably due to saturation of the submerged part of the sample and the slower hygric swelling of the upper part, which is outside the water and therefore it is only hygric swelling by capillarity. That defines two very different steps in the behavior of the rock during the experiment. In general, the whole vertical movement is expected to have a positive slope, as happens in $\mathrm{C} 1$ sample, which means that all movement increment was upwards and the rock keeps expanding during the whole experiment. However, in $\mathrm{C} 2$ sample a descending movement can be seen from minute 15 to 1.5 hours, approximately and it appears also for a small interval in sample C4. Some possible explanations could be given for such unexpected behavior. One of them is related to some rigid body rotations in the image plane during the sample hydration that may results in displacements in the upper part of the sample that are not due to swelling.

The studied sandstone presents different grain minerals and matrix that behaves differently in contact with water. Additionally, those grains have a random distribution in the sample and, when the ratio between the sizes of the sample and the grain size is high enough that distribution can be considered as homogeneous. However, if the ratio is low (as it is in our samples), the grain size takes importance and its location in the sample becomes relevant. This sandstone has a clayrich matrix that enhances the hygric swelling and that may be locally heterogeneously distributed in the studied small samples.

That uneven clay distribution could lead to an asymmetric swelling with respect to a vertical plane perpendicular to the image plane. That asymmetry will impose an ascendant vertical force that, if not compensated by the sample weight (due to its reduced size), will provoke a rotation or lateral movement of the sample. Moreover, the bottom side of the sample is non-perfectly flat due to local irregularities, that can act as a pivot for the rotation. Another possible explanation is related to the swelling dynamics and its relationship with environmental conditions, which were not controlled in this work.

The unexpected movement described in the previous paragraph was even more noticeable in the horizontal movement (figure 7 (right)). If the movement were due to only a swelling of the rock a positive slope should appear for the whole time. However, all slopes obtained for the horizontal movements at almost all times are negative, which means that the upper left sample corner is moving to the right, instead to the left, as expected. Under our point of view, this reinforces the fact that some rotations are happening. A rotation movement with respect to some point in the sample base will have a horizontal component much higher than the vertical one, therefore having a stronger influence in horizontal movements than in verticals, as it was observed in figure 7. The way to check if there was that rotation or not is to have at least both vertical sample boundaries inside the image, which leads to a pourer image resolution and losing the speckle type texture needed to perform DIC with accuracy. Therefore, one possible solution would be to use two cameras, one of them having the best resolution and the other one only devoted to measure rigid body movements.
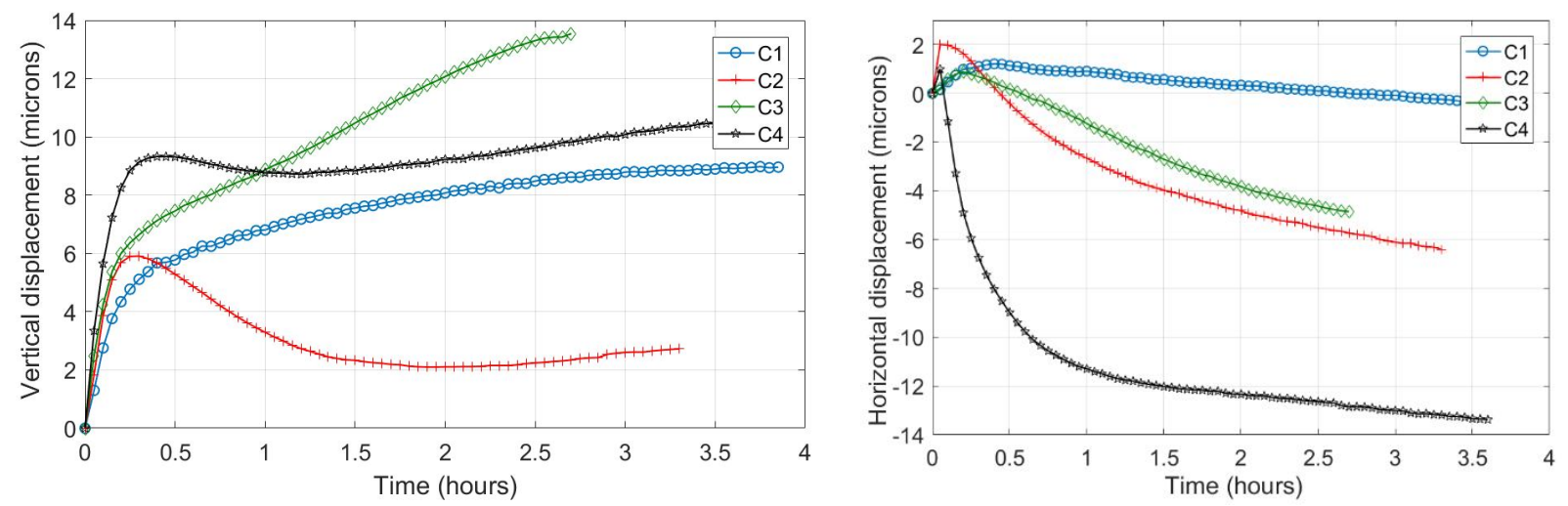

Figure 7. Vertical (left) and horizontal displacement (right) versus time for all samples measured by DIC.

As rigid solid movements could be involved in the movement measured from the sample corner, in principle, strain could not possible be determined using those data and the sample size. However, if we could measure vertical movements for two points separated a known vertical distance between them, a vertical strain still could be found. The same reasoning 
can be applied to the horizontal strain. Therefore, the displacements for 4 different ROIS were obtained (figure 8). For all those ROIS the same procedure described in the previous section was done.

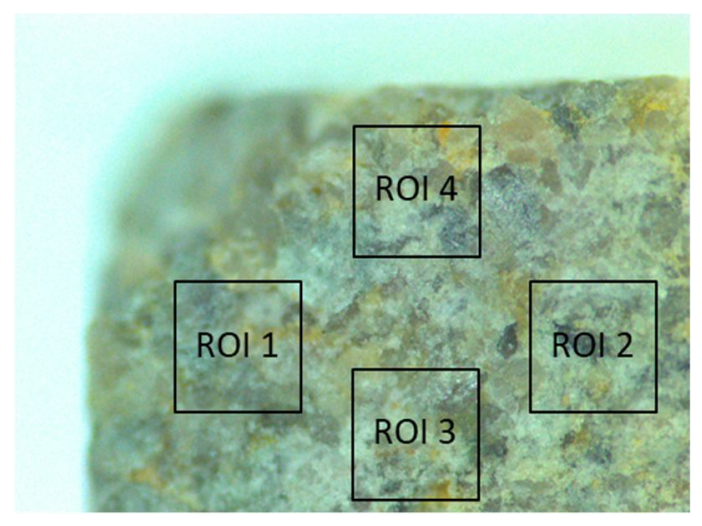

Figure 8. Four ROIs are taken in order to find the strain following equation (1). In this figure, ROIs taken for sample C2 are shown.

After calculation of all position ROIS, the strain was determined following the equation (1), where $\varepsilon_{x}$ and $\varepsilon_{y}$ are strains in vertical and horizontal directions, respectively, $L_{i}$ is position of ROI number $i$, and $d_{i-j}$ is distance from the center of ROI number $i$ to the center of ROI number $j$.

$$
\varepsilon_{x}=\frac{L_{1}-L_{2}}{d_{1-2}} \quad \varepsilon_{y}=\frac{L_{4}-L_{3}}{d_{4-3}}
$$

The results of strain calculations only gave some noisy results without any clear tendency. Several ROIs sizes and locations were tried in order to improve the results, but they were not conclusive in any significant manner. Under this negative result, our conclusion is that the image texture was good enough to DIC calculations taking a ROI size as that shown in figure 5, but not for the sizes shown in figure 8 . As rigid body rotations were not expected, image texture was initially planned to measure movement using almost the whole image, not the half part of it, as strain calculations following (1) force us. That is to say, the resolution was probably too high to use ROIs with the size in figure 8 . Those ROIs could not capture the texture of the stone, and the position of the individual components will gain importance in the correlation results. Therefore, movement perception would rely more in the relative brightness of the component rather than in the movement itself. This produced noisy results in all the ROIS that, when combined according to eq. (1) gave inconsistent curves.

In any case, and for the sake of obtaining a parameter that can be compared with those in the literature, we have obtained the strain from the general results depicted n Figure 7. We should consider that, although rotations are expected, they have small influence over the vertical strain. Consequently, we assume that the bottom part is not moving and then equation (1) is simplified to just divide vertical displacement by its total vertical length $(30 \mathrm{~mm})$. Figure 9 displays the vertical strain or vertical hygric swelling calculated through eq. (1). From these data we can see that the maximum value obtained in the first step of swelling is among 300 and $160 \mu \varepsilon\left(\times 10^{-6}\right)$, while its final value is between 300 and $350 \mu \varepsilon$. As sample $\mathrm{C} 2$ has a pronounced descending trend in the second step of swelling, its final strain cannot be considered. Values obtained for all samples in both swelling steps and mean values are presented in table 1 . They are comparable to similar sandstones in terms of mineralogical composition and porosity [1].

The curves obtained are expected to have the shape of a square root due to the capillary water movement. The imbibition kinetics of water in porous media was analyses theoretically by Washburn [9]. In his simple model, the porous medium is represented by a collection of parallel tubes, each having the same radius. The capillary imbibition kinetics shows two parts, clearly observed in samples $\mathrm{C} 1$ and C3: a first part that defines water adsorption, and a second part defining the saturation part. The slope of the curve during water adsorption is the water absorption coefficient that it is essentially equivalent to the sorptivity parameter in soil and material sciences [10]. 


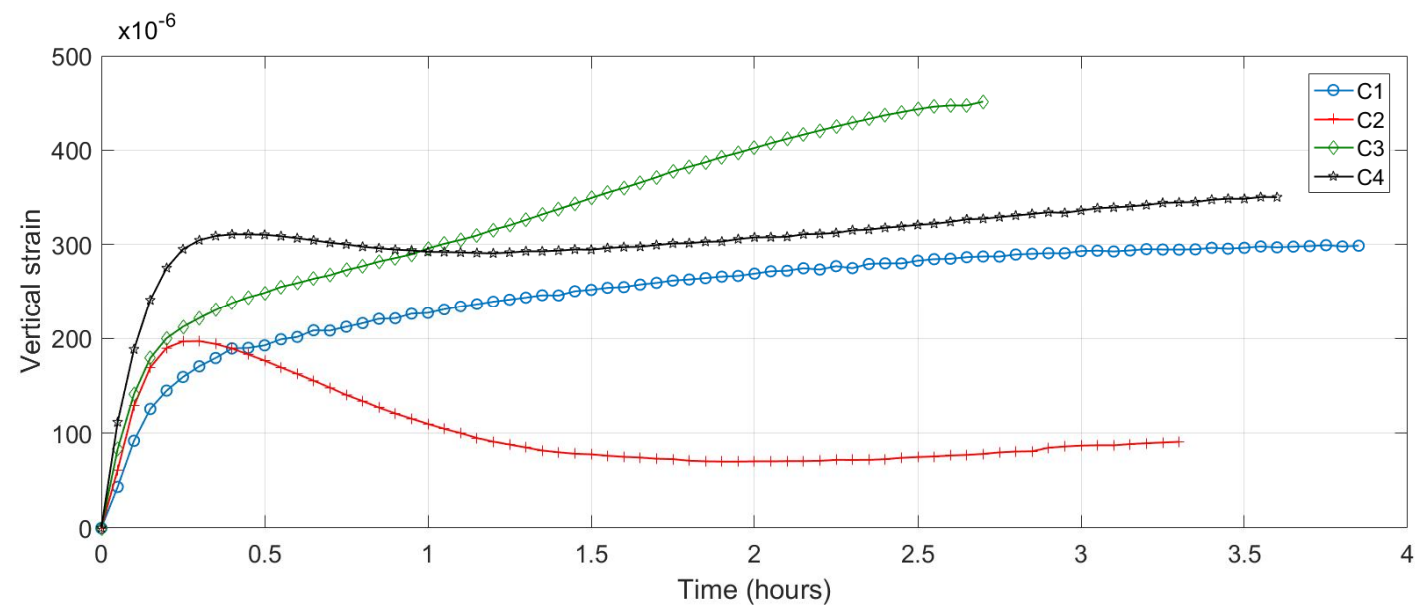

Figure 9. Vertical strains estimated from vertical movement and considering the whole vertical sample size.

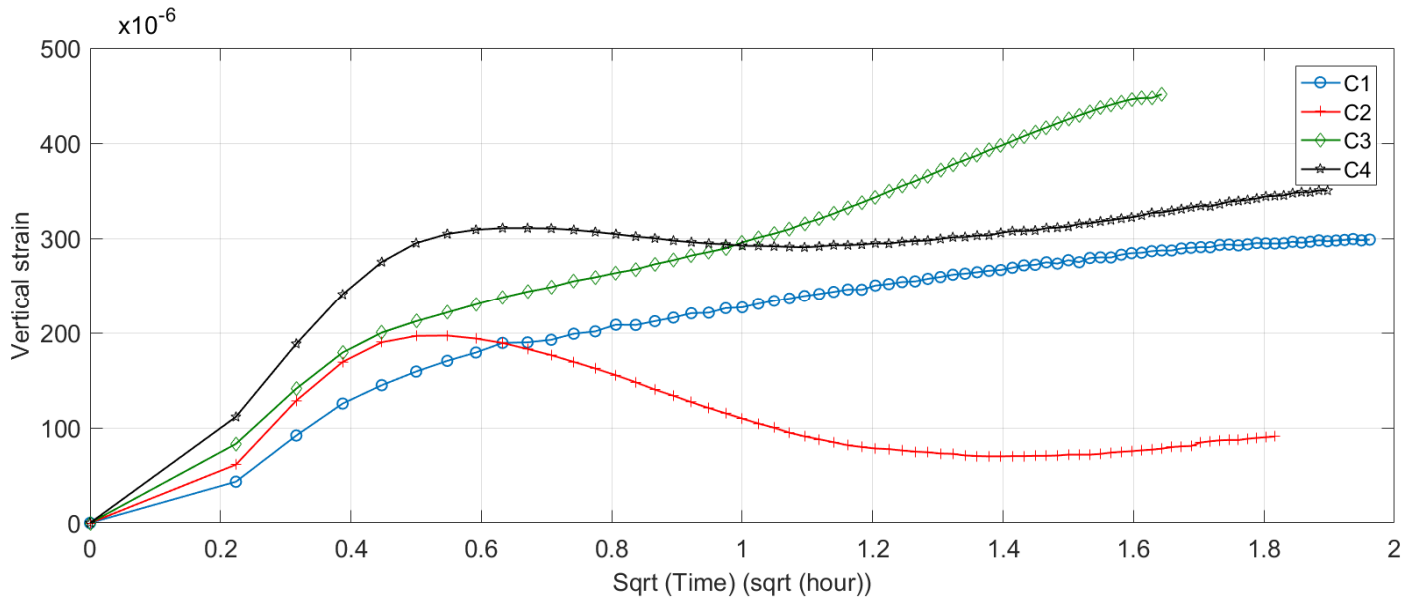

Figure 10. Vertical strains versus square root of time.

Table 1. Vertical strain in $\mu \varepsilon$ at final first step and at the end of the experiment.

\begin{tabular}{|c|c|c|}
\hline Sample & \multicolumn{2}{|c|}{ Vertical strain $\left(\mathbf{x 1 0}^{-6}\right)$} \\
\hline & First swelling step $(0.25$ hours $)$ & Final \\
\hline C1 & 159 & 299 \\
\hline C2 & 197 & --- \\
\hline C3 & 212 & 385 \\
\hline C4 & 295 & 350 \\
\hline Mean & 216 & 344 \\
\hline
\end{tabular}




\section{CONCLUSIONS}

Vertical and horizontal movements were measured in the upper left corner of some rock samples. The proposed method provided the displacement of this region of the stone, and, thus, stone dilation due to hygric swelling has been analyzed. Strain results have shown to be inconclusive, since it seems that some rigid body rotations could had happened during the second hydration phase. As those movements were not expected, image field was not wide enough to take smaller ROIS in order to subtract movements due to rotations than those measured. As vertical movement will be less affected by rotations, vertical movements were used in order to obtain vertical strains. Horizontal strains could not be calculated since horizontal movement will lead to high horizontal contractions that are not reasonable during a rock swelling. In order to have some information regarding the rigid body sample movement, a second camera could be used to record images in which, at least, two sample sides be inside of the image. With a whole view, the technique applied in [8] could be used also here to obtain a full description of the rock behavior.

\section{ACKNOWLEDGEMENTS}

This work has been supported by the Generalitat Valenciana and the European Social Fund (FSE) through the Recruitment of Predoctoral Research Staff ACIF/2018/211 included in the FSE Operational Program 2014-2020 of the Valencian Community.

\section{REFERENCES}

[1] Siegesmund S; Dürrast H. Physical and Mechanical Properties of Rocks. In: Siegesmund S, Snethlage R editors. Stone in Architecture, 4th ed. Springer-Verlag Berlin Heidelberg (2011) 97-225.

[2] Rawat, A; Baille, W; Tripathy, S; "Swelling behavior of compacted bentonite-sand mixture during water infliltration" Engineering Geology 257 (2019) 105141.

[3] Wasantha, P; Ranjith, P; Permata, G; Bing, D; "Damage evolution and deformation behavior of dry and saturated sandstones: Insights gleaned from optical measurements" Measurement 130 (2018) 8-17.

[4] Lee, S.S.; Jeong, G.Y.; "Effects of sample size on swelling and shrinkage of Larix kaempferi and Cryptomeria japonica as determined by digital caliper, image analysis, and digital image correlation (DIC)" Holzforschung 72:477-488

[5] Wang, L; Bornet, M; Yang, D; Héripré, E; Chanchole, S; Halphen, B; Pouya, A; Caldemaison, D; "Microstructural insight into the nonlinear swelling of argillaceous rocks" Engineering Geology 193 (2015) 435444

[6] https://www.gom.com/metrology-systems/aramis.html. Last time accessed on February, the 14th (2020)

[7] Ferrer, B.; Mas, D. "Parametric evaluation of errors using isolated dots for movement measurement by image cross-correlation" Sensors 18(2) (2018) 525

[8] Ferrer, B., Espinosa, J., Mas, D. "A method to measure small local strains in concrete surfaces using its natural texture and image cross-correlation" Structural Control and Health Monitoring 26(9) (2019) e2410.

[9] Washburn, E. W. (1921): The dynamics of capillary flow, Phys. Rev. 17, 273-283.

[10] Benavente, D., Lock, P., Garcia-del-Cura, M.A., Ordoñez, S. (2002): Predicting the capillary imbibition of porous rocks from microstructure. Trans Porous Media 49:59-76 